\title{
The Form Factor Program:
}

a Review and New Results -

\section{the Nested $S U(N)$ Off-Shell Bethe Ansatz ${ }^{\star}$}

\author{
Hratchya M. BABUJIAN ${ }^{\dagger}$, Angela FOERSTER $\ddagger$ and Michael KAROWSKI ${ }^{\S}$ \\ $\dagger$ Yerevan Physics Institute, Alikhanian Brothers 2, Yerevan, 375036, Armenia \\ E-mail: babujian@physik.fu-berlin.de \\ $\ddagger$ Instituto de Fúsica da UFRGS, Av. Bento Gonçalves 9500, Porto Alegre, RS - Brazil \\ E-mail: angela@if.ufrgs.br \\ $\S$ Theoretische Physik, Freie Universität Berlin, Arnimallee 14, 14195 Berlin, Germany \\ E-mail:karowski@physik.fu-berlin.de \\ URL: http://www.physik.fu-berlin.de/ ${ }^{\sim}$ karowski/
}

Received September 29, 2006, in final form November 16, 2006; Published online November 23, 2006

Original article is available at http://www.emis.de/journals/SIGMA/2006/Paper082/

\begin{abstract}
The purpose of the "bootstrap program" for integrable quantum field theories in $1+1$ dimensions is to construct explicitly a model in terms of its Wightman functions. In this article, this program is mainly illustrated in terms of the sinh-Gordon model and the $S U(N)$ Gross-Neveu model. The nested off-shell Bethe ansatz for an $S U(N)$ factorizing S-matrix is constructed. We review some previous results on sinh-Gordon form factors and the quantum operator field equation. The problem of how to sum over intermediate states is considered in the short distance limit of the two point Wightman function for the sinh-Gordon model.
\end{abstract}

Key words: integrable quantum field theory; form factors

2000 Mathematics Subject Classification: 81T08; 81T10; 81T40

\section{Introduction}

The bootstrap program to formulate particle physics in terms of the scattering data, i.e. in terms of the S-matrix goes back to Heisenberg [1] and Chew [2]. Remarkably, this approach works very well for integrable quantum field theories in $1+1$ dimensions $[3,4,5,6,8,7]$. The program does not start with any classical Lagrangian. Rather it classifies integrable quantum field theoretic models and in addition provides their explicit exact solutions in term of all Wightman functions. We achieve contact with the classical models only, when at the end we compare our exact results with Feynman graph (or other) expansions which are usually based on Lagrangians. However, there is no reason that the resulting quantum field theory is related to a classical Lagrangian.

One of the authors (M.K.) et al. [4] formulated the on-shell program i.e. the exact determination of the scattering matrix using the Yang-Baxter equations. The concept of generalized form factors was introduced by one of the authors (M.K.) et al. [8]. In this article consistency equations were formulated which are expected to be satisfied by these quantities. Thereafter this approach was developed further and studied in the context of several explicit models by Smirnov [9] who proposed the form factor equations $(i)-(v)$ (see below) as extensions of similar

${ }^{\star}$ This paper is a contribution to the Proceedings of the O'Raifeartaigh Symposium on Non-Perturbative and Symmetry Methods in Field Theory (June 22-24, 2006, Budapest, Hungary). The full collection is available at http://www.emis.de/journals/SIGMA/LOR2006.html 
formulae in the original article [8]. These formulae were then proven by two of the authors et al. [10]. In the present article we apply the form factor program for an $S U(N)$ invariant S-matrix (see [11]). The procedure is similar to that for the $Z(N)$ and $A(N-1)$ cases $[12,13]$ because the bound state fusions are similar in these three models. However, the algebraic structure of the form factors for the $S U(N)$ model is more intricate, because the S-matrix also describes backward scattering. We have to apply the nested "off-shell" ${ }^{1}$ Bethe ansatz, which was originally formulated by one of the authors (H.B.) $[14,15,16]$ to calculate correlation function in WZNW models (see also $[17,18]$ ).

Finally the Wightman functions are obtained by taking integrals and sums over intermediate states. The explicit evaluation of all these integrals and sums remains an open challenge for almost all models, except the Ising model [19, 20, 21, 7, 22]. In this article we discuss this problem for the examples of the sinh-Gordon model (see [23]). We investigate the short distance behavior of the two-point Wightman function of the exponentiated field.

\section{The "bootstrap program"}

The 'bootstrap program' for integrable quantum field theories in $1+1$-dimensions provides the solution of a model in term of all its Wightman functions. The result is obtained in three steps:

1. The S-matrix is calculated by means of general properties such as unitarity and crossing, the Yang-Baxter equations (which are a consequence of integrability) and the additional assumption of 'maximal analyticity'. This means that the two-particle S-matrix is an analytic function in the physical plane (of the Mandelstam variable $\left.\left(p_{1}+p_{2}\right)^{2}\right)$ and possesses only those poles there which are of physical origin. The only input which depends on the model is the assumption of a particle spectrum with an underlining symmetry. Typically there is a correspondence of fundamental representations with multiplets of particles. A classification of all S-matrices obeying the given properties is obtained.

2. Generalized form factors which are matrix elements of local operators

$$
\text { out }\left\langle p_{m}^{\prime}, \ldots, p_{1}^{\prime}|\mathcal{O}(x)| p_{1}, \ldots, p_{n}\right\rangle^{\text {in }}
$$

are calculated by means of the S-matrix. More precisely, the equations $(i)-(v)$ as listed in Section 3 are solved. These equations follow from LSZ-assumptions and again the additional assumption of 'maximal analyticity' [10].

3. The Wightman functions are obtained by inserting a complete set of intermediate states. In particular the two point function for a Hermitian operator $\mathcal{O}(x)$ reads

$$
\begin{aligned}
\langle 0|\mathcal{O}(x) \mathcal{O}(0)| 0\rangle= & \sum_{n=0}^{\infty} \frac{1}{n !} \int \cdots \int \frac{d p_{1} \cdots d p_{n}}{(2 \pi)^{n} 2 \omega_{1} \cdots 2 \omega_{n}} \\
& \times\left|\left\langle 0|\mathcal{O}(0)| p_{1}, \ldots, p_{n}\right\rangle^{\text {in }}\right|^{2} e^{-i x \sum p_{i}}
\end{aligned}
$$

Up to now a direct proof that these sums converge exists only for the scaling Ising model [19, 20, 21] and the non-unitary 'Yang-Lee' model [24].

Recently, Lechner [25] has shown that models with factorizing S-matrices exist within the framework of algebraic quantum field theory. For the algebraic approach to quantum field theories with factorizing S-matrices see the works of Schroer and Schroer-Wiesbrock [26, 27, 28]. A determinant representation for correlation functions of integrable models has been obtained by Korepin et al. [29, 30].

\footnotetext{
1 "Off-shell" in the context of the Bethe ansatz means that the spectral parameters in the algebraic Bethe ansatz state are not fixed by Bethe ansatz equations in order to get an eigenstate of a Hamiltonian, but they are integrated over.
} 


\section{Integrability}

Integrability in (quantum) field theories means that there exist infinitely many local conservation laws

$$
\partial_{\mu} J_{L}^{\mu}(t, x)=0 \quad(L= \pm 1, \pm 3, \ldots) .
$$

A consequence of such conservation laws in $1+1$ dimensions is that there is no particle production and the $\mathrm{n}$-particle S-matrix is a product of 2-particle S-matrices

$$
S^{(n)}\left(p_{1}, \ldots, p_{n}\right)=\prod_{i<j} S_{i j}\left(p_{i}, p_{j}\right) .
$$

If backward scattering occurs the 2-particle S-matrices will not commute and one has to specify the order. In particular for the 3-particle S-matrix there are two possibilities

$$
\mathbf{S}^{(3)}=\mathbf{S}_{12} \mathbf{S}_{13} \mathbf{S}_{23}=\mathbf{S}_{23} \mathbf{S}_{13} \mathbf{S}_{12}
$$

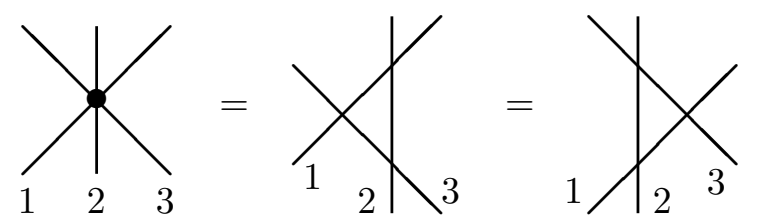

which yield the "Yang-Baxter Equation".

The two particle S-matrix is of the form

$$
S_{\alpha \beta}^{\beta^{\prime} \alpha^{\prime}}\left(\theta_{12}\right)=\searrow_{\alpha}^{\beta^{\prime}}
$$

where $\alpha, \beta$ etc denote the type of the particles and the rapidity difference $\theta_{12}=\theta_{1}-\theta_{2}>0$ is defined by $p_{i}=m_{i}\left(\cosh \theta_{i}, \sinh \theta_{i}\right)$. We also use the short hand notation $S_{12}\left(\theta_{12}\right)$. It satisfies unitarity

$$
S_{21}\left(\theta_{21}\right) S_{12}\left(\theta_{12}\right)=1: \quad \sum_{12}^{X}=\left.\right|_{1} \mid
$$

and crossing

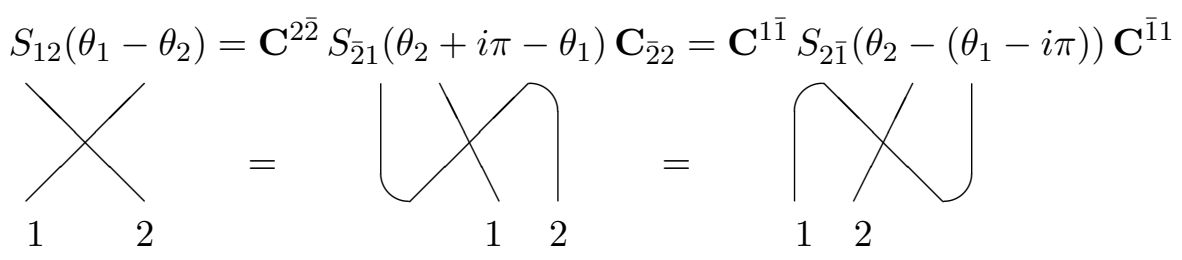

where $\mathbf{C}^{1 \overline{1}}$ and $\mathbf{C}_{1 \overline{1}}$ are charge conjugation matrices. We have introduced the following graphical rule, that a line changing the "time direction" also interchanges particles and anti-particles and changes the rapidity as $\theta \rightarrow \theta \pm i \pi$

$$
\mathbf{C}_{\alpha \bar{\beta}}=\theta \bigcap_{\alpha} \theta-i \pi, \quad \mathbf{C}^{\alpha \bar{\beta}}=\theta \bigcup^{\alpha} \theta+i \pi .
$$


Bound states: Let $\gamma$ be a bound state of particles $\alpha$ and $\beta$ with mass

$$
m_{\gamma}=\sqrt{m_{\alpha}^{2}+m_{\beta}^{2}+2 m_{\alpha} m_{\beta} \cos \eta}, \quad(0<\eta<\pi) .
$$

Then the 2 particle S-matrix has a pole such that

$$
\operatorname{Res}_{\theta=i \eta} S_{\alpha \beta}^{\beta^{\prime} \alpha^{\prime}}(\theta)=\Gamma_{\gamma}^{\beta^{\prime} \alpha^{\prime}} \Gamma_{\alpha \beta}^{\gamma}
$$

where $\eta$ is called the fusion angle and $\Gamma_{\alpha \beta}^{\gamma}$ is the 'bound state intertwiner' [31, 32]. The bound state S-matrix, that is the scattering matrix of the bound state (12) with a particle 3 , is obtained by the "bootstrap equation" [31]

$$
S_{(12) 3}\left(\theta_{(12) 3}\right) \Gamma_{12}^{(12)}=\Gamma_{12}^{(12)} S_{13}\left(\theta_{13}\right) S_{23}\left(\theta_{23}\right)
$$

where we use the usual short hand notation of matrices acting in the spaces corresponding to the particles 1, 2, 3 and (12).

Examples of integrable models in 1+1-dimensions (which we will consider in this review) are the sinh-Gordon model defined by the classical field equation

$$
\ddot{\varphi}(t, x)-\varphi^{\prime \prime}(t, x)+\frac{\alpha}{\beta} \sinh \beta \varphi(t, x)=0
$$

and the $\boldsymbol{S U}(\boldsymbol{N})$ Gross-Neveu model described by the Lagrangian

$$
\mathcal{L}=\bar{\psi} i \gamma \partial \psi+\frac{g^{2}}{2}\left((\bar{\psi} \psi)^{2}-\left(\bar{\psi} \gamma^{5} \psi\right)^{2}\right)
$$

where the Fermi fields form an $S U(N)$ multiplet.

Further integrable quantum field theories are: scaling $Z_{N}$-Ising models, nonlinear $\sigma$-models, $O(N)$ Gross-Neveu models, Toda models etc.

\section{The S-matrix}

The sinh-Gordon S-matrix is given by the analytic continuation $\beta \rightarrow i \beta$ of the sin-Gordon breather S-matrix [5]

$$
S(\theta)=\frac{\sinh \theta+i \sin \pi \nu}{\sinh \theta-i \sin \pi \nu} \quad \text { with } \quad-1 \leq \nu=\frac{-\beta^{2}}{8 \pi+\beta^{2}} \leq 0 .
$$

The model has the self-dual point at

$$
\nu=-\frac{1}{2} \quad \text { or } \quad \beta^{2}=4 \pi
$$


The $\boldsymbol{S U}(\boldsymbol{N})$ S-matrix: All solutions of $U(N)$-invariant S-matrix satisfying unitarity, crossing and the Yang-Baxter equation have been obtained in [33]. Following [34, 35], we adopt the view that in the $S U(N)$ Gross-Neveu model, the anti-particles are bound states of $N-1$ particles. This implies that there is no particle anti-particle backward scattering and that the $S U(N)$ S-matrix should be given by solution II of [33]. The scattering of the fundamental particles which form a multiplet corresponding to the vector representation of $S U(N)$ is (see [36, 37, 38] and [39] for $N=2$ )

$$
S_{\alpha \beta}^{\delta \gamma}(\theta)=\overbrace{\alpha}^{\delta} \gamma_{p_{1}}^{p_{4}} p_{p_{2}}^{p_{3}} \gamma_{\beta}^{\gamma}=\delta_{\alpha \gamma} \delta_{\beta \delta} b(\theta)+\delta_{\alpha \delta} \delta_{\beta \gamma} c(\theta)
$$

where due to Yang-Baxter $c(\theta)=-\frac{2 \pi i}{N \theta} b(\theta)$ holds and the highest weight amplitude is given as

$$
a(\theta)=b(\theta)+c(\theta)=-\frac{\Gamma\left(1-\frac{\theta}{2 \pi i}\right) \Gamma\left(1-\frac{1}{N}+\frac{\theta}{2 \pi i}\right)}{\Gamma\left(1+\frac{\theta}{2 \pi i}\right) \Gamma\left(1-\frac{1}{N}-\frac{\theta}{2 \pi i}\right)} .
$$

There is a bound state pole at $\theta=i \eta=2 \pi i / N$ in the antisymmetric tensor sector which agrees with Swieca's [34] picture that the bound state of $N-1$ particles is to be identified with the anti-particle. Similar as in the scaling $Z(N)$-Ising and $A(N-1)$-Toda models $[12,13]$ this will be used to construct the form factors in $S U(N)$ model [11].

\section{Form factors}

For a local operator $\mathcal{O}(x)$ the generalized form factors [8] are defined as

$$
F_{\alpha_{1} \ldots \alpha_{n}}^{\mathcal{O}}\left(\theta_{1}, \ldots, \theta_{n}\right)=\left\langle 0|\mathcal{O}(0)| p_{1}, \ldots, p_{n}\right\rangle_{\alpha_{1} \ldots \alpha_{n}}^{\text {in }}
$$

for $\theta_{1}>\cdots>\theta_{n}$. For other orders of the rapidities they are defined by analytic continuation. The index $\alpha_{i}$ denotes the type of the particle with momentum $p_{i}$. We also use the short notations $F_{\underline{\alpha}}^{\mathcal{O}}(\underline{\theta})$ or $F_{1 \ldots n}^{\mathcal{O}}(\underline{\theta})^{2}$.

For the $S U(N)$ Gross-Neveu model $\alpha$ denotes the types of particles belonging to all fundamental representations of $S U(N)$ with dimension $\left(\begin{array}{c}N \\ r\end{array}\right), r=1, \ldots, N-1$. In most formulae we restrict $\alpha=1, \ldots, N$ to the multiplet of the vector representation. Similar as for the S-matrix, 'maximal analyticity' for generalized form factors means again that they are meromorphic and all poles in the 'physical strips' $0 \leq \operatorname{Im} \theta_{i} \leq \pi$ have a physical interpretation. Together with the usual LSZ-assumptions [40] of local quantum field theory the following form factor equations can be derived:

(i) The Watson's equations describe the symmetry property under the permutation of both, the variables $\theta_{i}, \theta_{j}$ and the spaces $i, j=i+1$ at the same time

$$
F_{\ldots i j \ldots}^{\mathcal{O}}\left(\ldots, \theta_{i}, \theta_{j}, \ldots\right)=F_{\ldots j i \ldots}^{\mathcal{O}}\left(\ldots, \theta_{j}, \theta_{i}, \ldots\right) S_{i j}\left(\theta_{i j}\right)
$$

for all possible arrangements of the $\theta$ 's.

(ii) The crossing relation which implies a periodicity property under the cyclic permutation of the rapidity variables and spaces

$$
\begin{aligned}
\text { out }, \overline{1} & \left\langle p_{1}|\mathcal{O}(0)| p_{2}, \ldots, p_{n}\right\rangle_{2 \ldots n}^{\text {in,conn. }} \\
& =\mathbf{C}^{\overline{1} 1} \sigma_{1}^{\mathcal{O}} F_{1 \ldots n}^{\mathcal{O}}\left(\theta_{1}+i \pi, \theta_{2}, \ldots, \theta_{n}\right)=F_{2 \ldots n 1}^{\mathcal{O}}\left(\theta_{2}, \ldots, \theta_{n}, \theta_{1}-i \pi\right) \mathbf{C}^{1 \overline{1}}
\end{aligned}
$$

\footnotetext{
${ }^{2}$ The later means the co-vector in a tensor product space with the components $F_{\underline{\alpha}}^{\mathcal{O}}$.
} 
where $\sigma_{\alpha}^{\mathcal{O}}$ takes into account the statistics of the particle $\alpha$ with respect to $\mathcal{O}$ (e.g., $\sigma_{\alpha}^{\mathcal{O}}=-1$ if $\alpha$ and $\mathcal{O}$ are both fermionic, these numbers can be more general for anyonic or order and disorder fields, see [13]). For the charge conjugation matrix $\mathbf{C}^{\overline{1} 1}$ we refer to (3).

(iii) There are poles determined by one-particle states in each sub-channel given by a subset of particles of the state in (8).

In particular the function $F_{\underline{\alpha}}^{\mathcal{O}}(\underline{\theta})$ has a pole at $\theta_{12}=i \pi$ such that

$$
\underset{\theta_{12}=i \pi}{\operatorname{Res}} F_{1 \ldots n}^{\mathcal{O}}\left(\theta_{1}, \ldots, \theta_{n}\right)=2 i \mathbf{C}_{12} F_{3 \ldots n}^{\mathcal{O}}\left(\theta_{3}, \ldots, \theta_{n}\right)\left(\mathbf{1}-\sigma_{2}^{\mathcal{O}} S_{2 n} \cdots S_{23}\right) .
$$

(iv) If there are also bound states in the model the function $F_{\alpha}^{\mathcal{O}}(\underline{\theta})$ has additional poles. If for instance the particles 1 and 2 form a bound state (12), there is a pole at $\theta_{12}=i \eta$ $(0<\eta<\pi)$ such that

$$
\underset{\theta_{12}=\eta}{\operatorname{Res}} F_{12 \ldots n}^{\mathcal{O}}\left(\theta_{1}, \theta_{2}, \ldots, \theta_{n}\right)=F_{(12) \ldots n}^{\mathcal{O}}\left(\theta_{(12)}, \ldots, \theta_{n}\right) \sqrt{2} \Gamma_{12}^{(12)}
$$

where the bound state intertwiner $\Gamma_{12}^{(12)}$ is defined by (4) (see [31, 32]).

$(v)$ Naturally, since we are dealing with relativistic quantum field theories we finally have

$$
F_{1 \ldots n}^{\mathcal{O}}\left(\theta_{1}+\mu, \ldots, \theta_{n}+\mu\right)=e^{s \mu} F_{1 \ldots n}^{\mathcal{O}}\left(\theta_{1}, \ldots, \theta_{n}\right)
$$

if the local operator transforms under Lorentz transformations as $F^{\mathcal{O}} \rightarrow e^{s \mu} F^{\mathcal{O}}$ where $s$ is the "spin" of $\mathcal{O}$.

The properties $(i)-(i v)$ may be depicted as
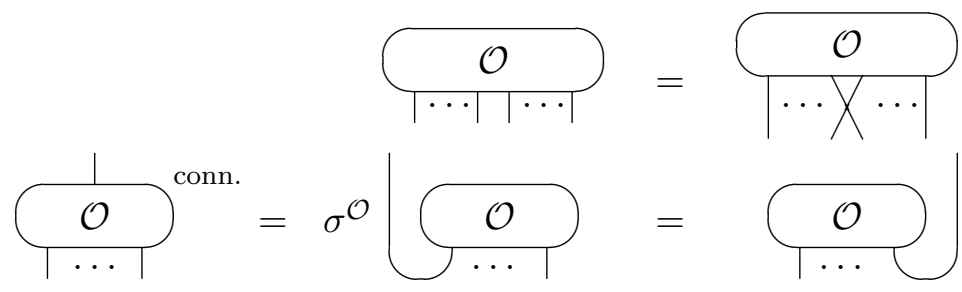

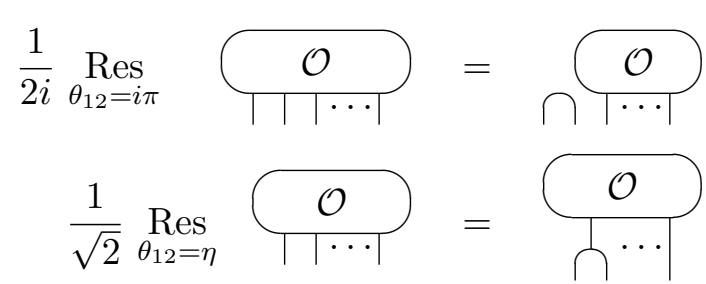

These equations have been proposed by Smirnov [9] as generalizations of equations derived in the original articles $[8,7,41]$. They have been proven [10] by means of the LSZ-assumptions and 'maximal analyticity'.

We will now provide a constructive and systematic way of how to solve the equations $(i)-(v)$ for the co-vector valued function $F_{1 \ldots n}^{\mathcal{O}}$ once the scattering matrix is given.

\subsection{Two-particle form factors}

For the two-particle form factors the form factor equations are easily understood. The usual assumptions of local quantum field theory yield

$$
\left\langle 0|\mathcal{O}(0)| p_{1}, p_{2}\right\rangle^{\text {in/out }}=F\left(\left(p_{1}+p_{2}\right)^{2} \pm i \varepsilon\right)=F\left( \pm \theta_{12}\right)
$$


where the rapidity difference is defined by $p_{1} p_{2}=m^{2} \cosh \theta_{12}$. For integrable theories one has particle number conservation which implies (for any eigenstate of the two-particle S-matrix)

$$
\left\langle 0|\mathcal{O}(0)| p_{1}, p_{2}\right\rangle^{\text {in }}=\left\langle 0|\mathcal{O}(0)| p_{2}, p_{1}\right\rangle^{\text {out }} S\left(\theta_{12}\right) .
$$

Crossing (10) means

$$
\left\langle p_{1}|\mathcal{O}(0)| p_{2}\right\rangle=F\left(i \pi-\theta_{12}\right)
$$

where for one-particle states the in- and out-states coincide. Therefore Watson's equations follow

$$
\begin{aligned}
& F(\theta)=F(-\theta) S(\theta), \\
& F(i \pi-\theta)=F(i \pi+\theta) .
\end{aligned}
$$

For general theories Watson's [42] equations only hold below the particle production thresholds. However, for integrable theories there is no particle production and therefore they hold for all complex values of $\theta$. It has been shown [8] that these equations together with "maximal analyticity" have a unique solution.

As an example we write the sinh-Gordon [8] and the (highest weight) $S U(N)$ form factor function [11]

$$
\begin{aligned}
& F^{S H G}(\theta)=\exp \int_{0}^{\infty} \frac{d t}{t \sinh t}\left(\frac{\cosh \left(\frac{1}{2}+\nu\right) t}{\cosh \frac{1}{2} t}-1\right) \cosh t\left(1-\frac{\theta}{i \pi}\right) \\
& F^{S U(N)}(\theta)=c \exp \int_{0}^{\infty} \frac{d t}{t \sinh ^{2} t} e^{\frac{t}{N}} \sinh t\left(1-\frac{1}{N}\right)\left(1-\cosh t\left(1-\frac{\theta}{i \pi}\right)\right)
\end{aligned}
$$

which are the minimal solution of (14) with $S^{S H G}(\theta)$ as given by $(5)$ and $S^{S U(N)}(\theta)=a(\theta)$ as given by (7), respectively.

\subsection{The general form factor formula}

As usual [8] we split off the minimal part and write the form factor for $\mathrm{n}$ particles as

$$
F_{\alpha_{1} \ldots \alpha_{n}}^{\mathcal{O}}\left(\theta_{1}, \ldots, \theta_{n}\right)=K_{\alpha_{1} \ldots \alpha_{n}}^{\mathcal{O}}(\underline{\theta}) \prod_{1 \leq i<j \leq n} F\left(\theta_{i j}\right) .
$$

By means of the following "off-shell Bethe ansatz" for the (co-vector valued) K-function

$$
K_{\alpha_{1} \ldots \alpha_{n}}^{\mathcal{O}}(\underline{\theta})=\int_{\mathcal{C}_{\underline{\theta}}} d z_{1} \cdots \int_{\mathcal{C}_{\underline{\theta}}} d z_{m} h(\underline{\theta}, \underline{z}) p^{\mathcal{O}}(\underline{\theta}, \underline{z}) \Psi_{\alpha_{1} \ldots \alpha_{n}}(\underline{\theta}, \underline{z})
$$

we transform the complicated form factor equations $(i)-(v)$ into simple ones for the p-functions which are scalar and polynomials in $e^{ \pm z_{i}}$. The "off-shell Bethe ansatz" state $\Psi_{\alpha_{1} \ldots \alpha_{n}}(\underline{\theta}, \underline{z})$ is obtained as a product of S-matrix elements and the integration contour $\mathcal{C}_{\underline{\theta}}$ depends on the model (see below for the $S U(N)$-model). The scalar functions

$$
\begin{aligned}
& h(\underline{\theta}, \underline{z})=\prod_{i=1}^{n} \prod_{j=1}^{m} \phi\left(\theta_{i}-z_{j}\right) \prod_{1 \leq i<j \leq m} \tau\left(z_{i}-z_{j}\right), \\
& \tau(z)=\frac{1}{\phi(z) \phi(-z)}
\end{aligned}
$$

depend on $S(\theta)$ only (see (25) below), i.e. on the S-matrix, whereas the $p$-function $p^{\mathcal{O}}(\underline{\theta}, \underline{z})$ depends on the operator. 


\section{The $S U(N)$ form factors}

The form factors for $\mathrm{n}$ fundamental particles (of the vector representation of $S U(N)$ ) are given by (17)-(19) where the "nested Bethe ansatz" is needed. This means that $\Psi$ is of the form

$$
\Psi_{\alpha_{1} \ldots \alpha_{n}}(\underline{\theta}, \underline{z})=L_{\beta_{1} \ldots \beta_{m}}(\underline{z}) \Phi_{\alpha_{1} \ldots \alpha_{n}}^{\beta_{1} \ldots \beta_{m}}(\underline{\theta}, \underline{z})
$$

where the indices $\alpha_{i}$ take the values $\alpha=1, \ldots, N$ and the summations run over $\beta_{i}=2, \ldots, N$. The "off-shell Bethe ansatz" state $\Phi_{\alpha_{1} \ldots \alpha_{n}}^{\beta_{1} \ldots \beta_{m}}(\underline{\theta}, \underline{z})$ is obtained using the techniques of the algebraic Bethe ansatz as follows.

We consider a state with $n$ particles and define the monodromy matrix

$$
T_{1 \ldots n, 0}\left(\underline{\theta}, \theta_{0}\right)=S_{10}\left(\theta_{10}\right) \cdots S_{n 0}\left(\theta_{n 0}\right)=\begin{array}{l|l|l} 
& \cdots & \\
\hline 1 & & n
\end{array}
$$

as a matrix acting in the tensor product of the "quantum space", a space of $n$ particles (with respect to their quantum numbers) $V^{1 \ldots n}=V^{1} \otimes \cdots \otimes V^{n}$ and the "auxiliary space" $V^{0}$. All vector spaces $V^{i}$ are isomorphic to a space $V$ whose basis vectors label all kinds of particles. Here we consider $V \cong \mathbb{C}^{N}$ as the space of the vector representation of $S U(N)$. The Yang-Baxter algebra relation for the S-matrix yields

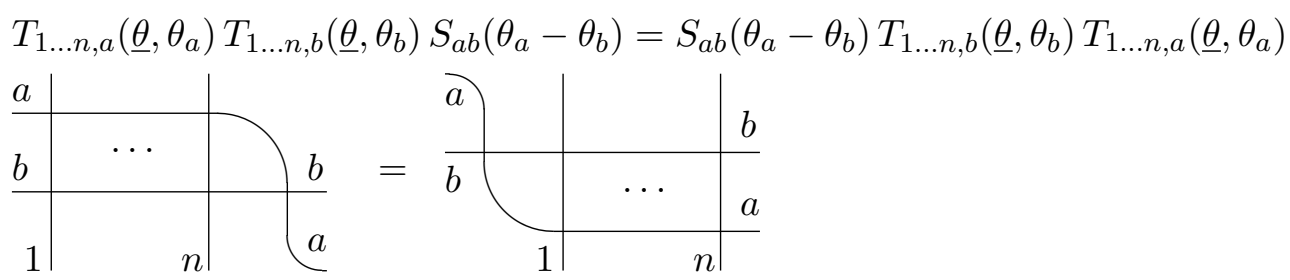

which in turn implies the basic algebraic properties of the sub-matrices $A, B, C, D$ with respect to the auxiliary space defined by

$$
T_{1 \ldots n, 0}(\underline{\theta}, z) \equiv\left(\begin{array}{cc}
A_{1 \ldots n}(\underline{\theta}, z) & B_{1 \ldots ., \beta}(\underline{\theta}, z) \\
C_{1 \ldots n}^{\beta}(\underline{\theta}, z) & D_{1 \ldots n, \beta}^{\beta^{\prime}}(\underline{\theta}, z)
\end{array}\right), \quad 2 \leq \beta, \beta^{\prime} \leq N .
$$

The basic Bethe ansatz co-vectors $\Phi$ of equation (20) are obtained by an application of the operators $C$ to a "pseudo-vacuum" state

$$
\Phi_{1 \ldots n}^{\underline{\beta}}(\underline{\theta}, \underline{z})=\Omega_{1 \ldots n} C_{1 \ldots n}^{\beta_{m}}\left(\underline{\theta}, z_{m}\right) \cdots C_{1 \ldots n}^{\beta_{1}}\left(\underline{\theta}, z_{1}\right) .
$$

This may be depicted as

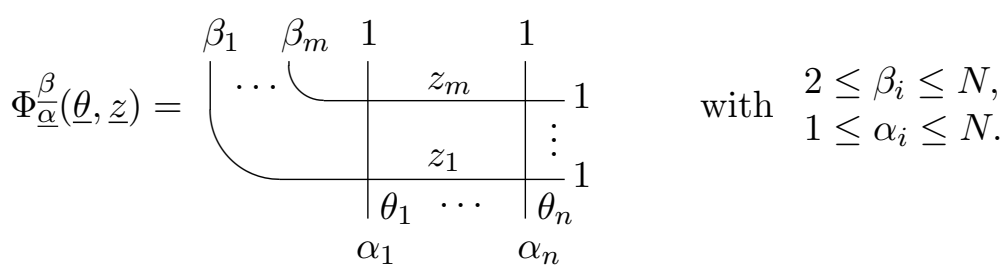

It means that $\Phi_{\underline{\alpha}}(\underline{\beta}, \underline{z})$ is a product of S-matrix elements as given by the picture where at all crossing points of lines there is an S-matrix (6) and the sum over all indices of internal lines is to be taken. The "pseudo-vacuum" is the highest weight co-vector (with weight $w=(n, 0, \ldots, 0)$ )

$$
\Omega_{1 \ldots n}=e(1) \otimes \cdots \otimes e(1)
$$


where the unit vectors $e(\alpha)(\alpha=1, \ldots, N)$ correspond to the particle of type $\alpha$ which belong to the vector representation of $S U(N)$. The pseudo-vacuum basic vector satisfies

$$
\begin{aligned}
& \Omega_{1 \ldots n} B_{1 \ldots n}^{\beta}(\underline{\theta}, z)=0 \\
& \Omega_{1 \ldots n} A_{1 \ldots n}(\underline{\theta}, z)=\prod_{i=1}^{n} a\left(\theta_{i}-z\right) \Omega_{1 \ldots n}, \\
& \Omega_{1 \ldots n} D_{1 \ldots n, \beta}^{\beta^{\prime}}(\underline{\theta}, z)=\delta_{\beta}^{\beta^{\prime}} \prod_{i=1}^{n} b\left(\theta_{i}-z\right) \Omega_{1 \ldots n} .
\end{aligned}
$$

The amplitudes of the scattering matrices are given by equation (6). The technique of the nested Bethe ansatz means that for the co-vector valued function $L_{\beta_{1} \ldots \beta_{m}}(\underline{z})$ in (20) one makes the second level Bethe ansatz. This ansatz is of the same form as (18) only that the range of the indices is reduced by 1 . Iterating this nesting procedure one finally arrives at a scalar function. The integration contour $\mathcal{C}_{\underline{\theta}}$ depends on the $\underline{\theta}$ and is depicted in Fig. 1.

$$
\begin{aligned}
& \text { - } \theta_{n}+2 \pi i\left(1-\frac{1}{N}\right) \quad \bullet \theta_{2}+2 \pi i\left(1-\frac{1}{N}\right) \stackrel{\bullet}{\theta_{1}+2 \pi i\left(1-\frac{1}{N}\right)}
\end{aligned}
$$



$$
\begin{aligned}
& \longrightarrow \quad \theta_{\bullet \theta_{n}-2 \pi i}^{\longrightarrow} \stackrel{\bullet \theta_{1}-2 \pi i}{\longrightarrow}
\end{aligned}
$$

Figure 1. The integration contour $C_{\underline{\theta}}$. The bullets belong to poles of the integrand resulting from $a\left(\theta_{i}-z_{j}\right) \phi\left(\theta_{i}-z_{j}\right)$ and the small open circles belong to poles originating from $b\left(\theta_{i}-z_{j}\right)$ and $c\left(\theta_{i}-z_{j}\right)$.

Swieca's picture is that the bound state of $N-1$ fundamental particles is to be identified with the anti-particle and lead together with the form factor recursion relations $(i i i)+(i v)$ to the equation for the function $\phi(z)$ (see $[13,11])$

$$
\prod_{k=0}^{N-2} \phi(z+k i \eta) \prod_{k=0}^{N-1} F(z+k i \eta)=1, \quad \eta=\frac{2 \pi}{N}
$$

with the solution

$$
\phi(\theta)=\Gamma\left(\frac{\theta}{2 \pi i}\right) \Gamma\left(1-\frac{1}{N}-\frac{\theta}{2 \pi i}\right) .
$$

In [11] it is shown that the form factors given by (17) and (18) satisfy the form factor equations $(i)-(v)$ if some simple equations for the p-function are satisfied. We note that form factors of this model were also calculated in [9, 43, 44] using other techniques.

\section{Locality}

Let us discuss a further property of the form factors which is directly related to the nature of the operators, that is locality. In fact, this property touches the very heart and most central 
concepts of relativistic quantum field theory, like Einstein causality and Poincaré covariance, which are captured in local field equations and commutation relations.

So far we have presented a formulation of a quantum field theory, which starts from a particle picture. It is basically possible to obtain the particle picture from the field formulation by means of the LSZ-reduction formalism. The reverse problem, namely of how to reconstruct the entire field content, or at least part of it, from the scattering theory is in general still an outstanding challenge. Besides this classification issue there remains also the general question if the operators which are related to the solutions of $(i)-(v)$ are genuinely local, meaning that they (anti)-commute for space-like separations with themselves. It has been shown that $(i)-(v)$ (see e.g. [9, 13]) imply

$$
\text { in }\langle\phi|[\mathcal{O}(x), \mathcal{O}(y)]| \psi\rangle^{i n}=0 \quad \text { for } \quad(x-y)^{2}<0
$$

for all matrix elements. Note that commutation rules such as (26) hold if the statistics factors in $(i)-(v)$ are trivial, $\sigma^{\mathcal{O}}=1$. However, for more general statistics factors there are also fermionic, anyonic and even order-disorder commutation rules as for example for the scaling $Z_{N}$-Ising model (see [13]).

\section{Examples of operators and their $p$-functions for the sinh-Gordon model}

Since there is no backward scattering the Bethe ansatz in (18) is trivial, the integrations can be performed [45] and the n-particle $K$-function may be written as

$$
K_{n}^{\mathcal{O}}(\underline{\theta})=\sum_{l_{1}=0}^{1} \cdots \sum_{l_{n}=0}^{1}(-1)^{\sum l_{i}} \prod_{i<j}\left(1+\left(l_{i}-l_{j}\right) \frac{i \sin \pi \nu}{\sinh \theta_{i j}}\right) p_{n}^{\mathcal{O}}(\underline{\theta}, \underline{l}) .
$$

For several cases the correspondence between local operators and their p-functions have been proposed in $[45]^{3}$. Here we provide three examples:

1. The normal ordered exponential of the field (see also [48])

$$
\mathcal{O}(x)=: e^{\gamma \varphi(x)}: \quad \leftrightarrow p(\underline{\theta}, \underline{l})=\left(\frac{2}{F(i \pi) \sin \pi \nu}\right)^{\frac{n}{2}} \prod_{i=1}^{n} e^{\pi \nu \frac{\gamma}{\beta}(-1)^{l_{i}}},
$$

2. Expanding the last relation with respect to $\gamma$ one obtains the $p$-functions for normal ordered powers : $\varphi(x)^{N}:$ in particular for $N=1$

$$
: \varphi(x): \leftrightarrow p(\underline{\theta}, \underline{l})=\frac{\pi \nu}{\beta}\left(\frac{2}{F(i \pi) \sin \pi \nu}\right)^{\frac{n}{2}} \sum_{i=1}^{n}(-1)^{l_{i}},
$$

which yields (for $n=1$ ) the 'wave function renormalization constant'

$$
Z^{\varphi}=\langle 0|\varphi(0)| p\rangle^{2}=\frac{8 \pi^{2} \nu^{2}}{-F^{S H G}(i \pi) \beta^{2} \sin \pi \nu}
$$

(see also [8]).

3. The higher conserved currents $J_{L}^{\mu}(x)$ which are typical for integrable quantum field theories

$$
J_{L}^{ \pm}(x) \leftrightarrow \pm N_{n}^{\left(J_{L}\right)} \sum_{i=1}^{n} e^{ \pm \theta_{i}} \sum_{i=1}^{n} e^{L\left(\theta_{i}-\frac{i \pi}{2}\left(1-(-1)^{l_{i} \nu}\right)\right)}
$$

\footnotetext{
${ }^{3}$ Sinh-Gordon form factors have been presented before in a different form in [46, 47].
} 


\section{Quantum sinh-Gordon field operator equation}

Using (28) and (29) one finds [49, 32, 45] that the quantum sinh-Gordon field equation

$$
\square \varphi(x)+\frac{\alpha}{\beta}: \sinh \beta \varphi:(x)=0
$$

holds for all matrix elements, if the "bare" mass $\sqrt{\alpha}$ is related to the renormalized mass by

$$
\alpha=m^{2} \frac{\pi \nu}{\sin \pi \nu}
$$

where $m$ is the physical mass of the fundamental boson. The result may be checked in perturbation theory by Feynman graph expansions. In particular in lowest order the relation between the bare and the renormalized mass (31) had already been calculated in the original article [8]. The result is

$$
m^{2}=\alpha\left(1-\frac{1}{6}\left(\frac{\beta^{2}}{8}\right)^{2}+O\left(\beta^{6}\right)\right)
$$

which agrees with the exact formula above. The factor $\frac{\pi \nu}{\sin \pi \nu}$ in (31) modifies the classical equation and has to be considered as a quantum correction. The proof of the quantum field equation (30) can be found in [45].

\section{Wightman functions}

As the simplest case we consider the two-point function of two local scalar operators $\mathcal{O}(x)$ and $\mathcal{O}^{\prime}(x)$

$$
w(x)=\left\langle 0\left|\mathcal{O}(x) \mathcal{O}^{\prime}(0)\right| 0\right\rangle .
$$

\section{Summation over all intermediate states}

Inserting a complete set of in-states we may write

$$
\begin{aligned}
w(x)= & \sum_{n=0}^{\infty} \frac{1}{n !} \int \frac{d p_{1}}{2 \pi 2 \omega_{1}} \cdots \int \frac{d p_{n}}{2 \pi 2 \omega_{n}} e^{-i x\left(p_{1}+\cdots+p_{n}\right)} \\
& \times\left\langle 0|\mathcal{O}(0)| p_{1}, \ldots, p_{n}\right\rangle^{\text {in in }\left\langle p_{n}, \ldots, p_{1}\left|\mathcal{O}^{\prime}(0)\right| 0\right\rangle} \\
= & \sum_{n=0}^{\infty} \frac{1}{n !} \int d \theta_{1} \cdots \int d \theta_{n} e^{-i x \sum p_{i}} g_{n}(\underline{\theta}) .
\end{aligned}
$$

We have introduced the functions

$$
\begin{aligned}
g_{n}(\underline{\theta}) & =\frac{1}{(4 \pi)^{n}}\left\langle 0|\mathcal{O}(0)| p_{1}, \ldots, p_{n}\right\rangle^{\text {in in }}\left\langle p_{n}, \ldots, p_{1}\left|\mathcal{O}^{\prime}(0)\right| 0\right\rangle \\
& =\frac{1}{(4 \pi)^{n}} F^{\mathcal{O}}\left(\theta_{1}, \ldots, \theta_{n}\right) F^{\mathcal{O}^{\prime}}\left(\theta_{n}+i \pi, \ldots, \theta_{1}+i \pi\right) .
\end{aligned}
$$

where crossing has been used. In particular we consider exponentials of a scalar bose field

$$
\mathcal{O}^{(\prime)}(x)=: e^{i \gamma^{(\prime)} \varphi(x)}:
$$

where $: \cdots$ : means normal ordering with respect to the physical vacuum and which amounts to the condition

$$
\left\langle 0\left|: e^{i \gamma^{(\prime)} \varphi(x)}:\right| 0\right\rangle=1
$$

and therefore $g_{0}=1$ holds. 
The Log of the two-point function For specific operators like exponentials of bose fields it might be convenient (see below) to consider a resummation of the sum in (32). For $g_{0}=1$ we may write (see also [50])

$$
\begin{aligned}
w(x) & =1+\sum_{n=1}^{\infty} \frac{1}{n !} \int d \theta_{1} \cdots \int d \theta_{n} e^{-i x \sum p_{i}} g_{n}(\underline{\theta}) \\
& =\exp \sum_{n=1}^{\infty} \frac{1}{n !} \int d \theta_{1} \cdots \int d \theta_{n} e^{-i x \sum p_{i}} h_{n}(\underline{\theta}) .
\end{aligned}
$$

It is well known that the functions $g_{n}$ and $h_{n}$ are related by the cummulant formula

$$
g_{I}=\sum_{I_{1} \cup \cdots \cup I_{k}=I} h_{I_{1}} \cdots h_{I_{k}}
$$

where we use the short hand notation $g_{I}=g_{n}\left(\theta_{1}, \ldots, \theta_{n}\right)$ with $I=\{1, \ldots, n\}$. The relations for the $g$ 's and the $h$ 's may be depicted with $g=\square$ and $h=\square$ as

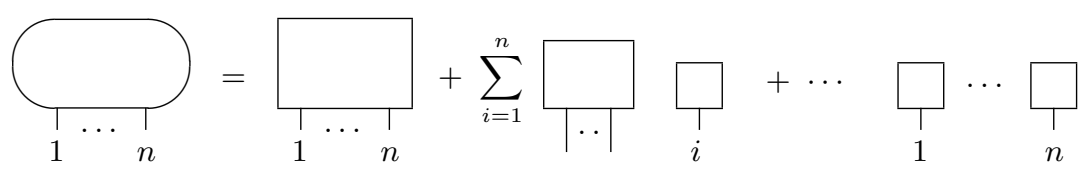

Thus as examples

$$
\begin{aligned}
& g_{1}=h_{1}, \\
& g_{12}=h_{12}+h_{1} h_{2}, \\
& g_{123}=h_{123}+h_{12} h_{3}+h_{13} h_{2}+h_{23} h_{1}+h_{1} h_{2} h_{3},
\end{aligned}
$$

Due to Lorentz invariance it is sufficient to consider the value $x=(-i \tau, 0)$. Let $\mathcal{O}(x)$ and $\mathcal{O}^{\prime}(x)$ be scalar operators. Then the functions $h_{n}(\underline{\theta})$ depend only on the rapidity differences. We use the formula for the modified Bessel function of the third kind

$$
i \Delta_{+}(x)=\langle 0|\varphi(x) \varphi(0)| 0\rangle=\frac{1}{4 \pi} \int d \theta e^{-\tau m \cosh \theta}=\frac{1}{2 \pi} K_{0}(m \tau)
$$

to perform one integration

$$
\begin{aligned}
\ln w(x) & =\sum_{n=1}^{\infty} \frac{1}{n !} \int d \theta_{1} \cdots \int d \theta_{n} e^{-\tau m \sum \cosh \theta_{i}} h_{n}(\underline{\theta}) \\
& =2 \sum_{n=1}^{\infty} \frac{1}{n !} \int d \theta_{1} \cdots \int d \theta_{n-1} h_{n}\left(\theta_{1}, \ldots, \theta_{n-1}, 0\right) K_{0}(m \tau \xi)
\end{aligned}
$$

with

$$
\xi^{2}=\left(\sum_{i=1}^{n-1} \cosh \theta_{i}+1\right)^{2}-\left(\sum_{i=1}^{n-1} \sinh \theta_{i}\right)^{2}
$$




\section{Short distance behavior $x \rightarrow 0$}

In order to perform the conformal limit of massive models one investigates the short distance behavior (see e.g. [51, 52, 50]). For small $\tau$ we use the expansion of the modified Bessel function of the third kind and obtain

$$
\begin{aligned}
\ln w(x)= & -2 \sum_{n=1}^{\infty} \frac{1}{n !} \int d \theta_{1} \ldots \int d \theta_{n-1} h_{n}\left(\theta_{1}, \ldots, \theta_{n-1}, 0\right) \\
& \times\left(\ln m \tau+\ln \xi+\gamma_{E}-\ln 2+O\left(\tau^{2} \ln \tau\right)\right)
\end{aligned}
$$

where $\gamma_{E}=0.5772 \ldots$ is Euler's or Mascheroni's constant. Therefore the two-point Wightman function has power-like behavior for short distances

$$
w(x) \approx C(m \tau)^{-4 \Delta} \quad \text { for } \quad \tau \rightarrow 0
$$

where the dimension is given by

$$
\Delta=\frac{1}{2} \sum_{n=1}^{\infty} \frac{1}{n !} \int d \theta_{1} \cdots \int d \theta_{n-1} h_{n}\left(\theta_{1}, \ldots, \theta_{n-1,0}\right)
$$

in case the integrals exist. This is true for the exponentials of bose fields $\mathcal{O}=: e^{\gamma \varphi(x)}:$ due to the asymptotic behavior for $\operatorname{Re} \theta_{1} \rightarrow \infty$

$$
\begin{aligned}
& F_{n}^{\mathcal{O}}\left(\theta_{1}, \theta_{2}, \ldots\right)=F_{1}^{\mathcal{O}}\left(\theta_{1}\right) F_{n-1}^{\mathcal{O}}\left(\theta_{2}, \ldots\right)+O\left(e^{-\theta_{1}}\right), \\
& g_{n}\left(\theta_{1}, \theta_{2}, \ldots, \theta_{n}\right)=g_{1} g_{n-1}\left(\theta_{2}, \ldots, \theta_{n}\right)+O\left(e^{-\left|\theta_{1}\right|}\right)
\end{aligned}
$$

(see e.g. [45]). Therefore the functions $h_{n}$ satisfy

$$
h_{n}(\underline{\theta})=O\left(e^{-\left|\theta_{i}\right|}\right) \quad \text { for } \quad \operatorname{Re} \theta_{i} \rightarrow \pm \infty .
$$

This follows when we distinguish the variable $\theta_{1}$ in the relation of the $g$ 's and the $h$ 's above and reorganize the terms on the right hand side as follows

$$
g_{I}=\sum_{1 \in J \subseteq I} h_{J} g_{I \backslash J} .
$$

The constant $C$ is obtained as

$$
C=\exp \left(-2 \sum_{n=1}^{\infty} \frac{1}{n !} \int d \theta_{1} \cdots \int d \theta_{n-1} h_{n}\left(\theta_{1}, \ldots, \theta_{n-1}, 0\right)\left(\ln \frac{1}{2} \xi+\gamma_{E}\right)\right)
$$

and it should be related to the vacuum expectation value $G=\langle 0|\mathcal{O}(x)| 0\rangle_{C}$ in the 'conformal normalization'

$$
C=m^{4 \Delta} G^{-2} .
$$

Such vacuum expectation value was calculated in [53] for the sine-Gordon model.

\section{Example: the sinh-Gordon model}

The dimension of the exponential of the field $\mathcal{O}(x)=: e^{\beta \varphi(x)}$ : for the sinh-Gordon model may be calculated in the 1- and 1+2-particle intermediate state approximation (see Fig. 2) as

$$
\begin{aligned}
\Delta_{1+2} & =\frac{1}{2}\left(h_{1}+\frac{1}{2 !} \int d \theta h_{2}(\theta, 0)+\cdots\right) \\
& =-\frac{\sin \pi \nu}{\pi F(i \pi)}+\left(\frac{\sin \pi \nu}{\pi F(i \pi)}\right)^{2} \int_{-\infty}^{\infty} d \theta(F(\theta) F(-\theta)-1)+\cdots .
\end{aligned}
$$




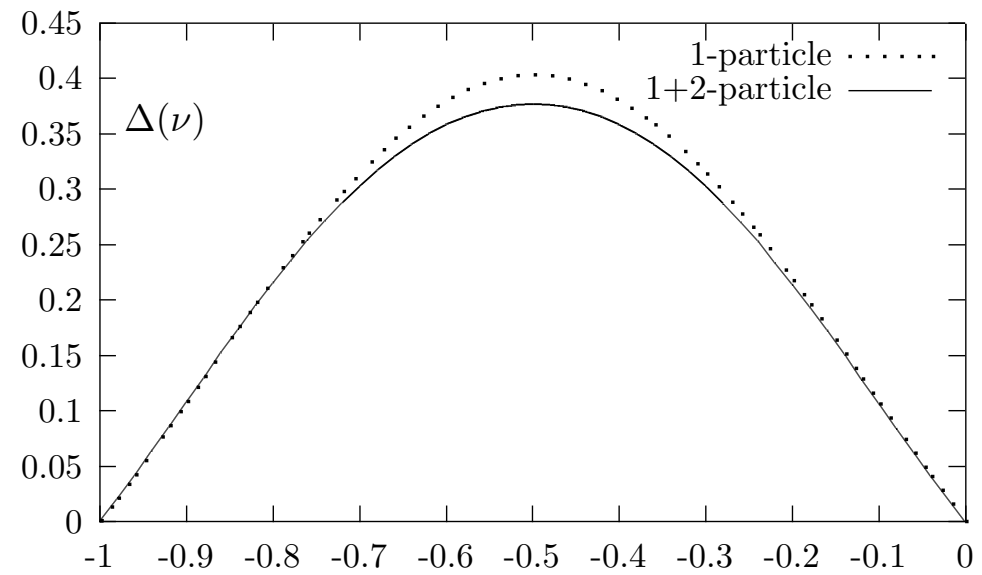

Figure 2. Dimension of an exponential of the field for the sinh-Gordon model: 1- and 1+2-particle intermediate state contributions.

The integral may be calculated exactly with the result [54]

$$
-\frac{\pi}{2} \sin \pi \nu F^{2}(i \pi)-\pi \frac{\cos \pi \nu-1}{\sin \pi \nu}+2\left(1-\frac{\pi \nu \cos \pi \nu}{\sin \pi \nu}\right) .
$$

In principle the higher particle intermediate state integrals may also be calculated, however, up to now we were not able to derive a general formula. For the scaling Ising model, however, this is possible. The constant $C$ in the approximation of 1-intermediate states is given as

$$
C_{1}=\exp \left(-2 h_{1}\left(\gamma_{E}-\ln 2\right)\right)=\exp \left(4 \frac{\sin \pi \nu}{\pi F(i \pi)}\left(\gamma_{E}-\ln 2\right)\right) .
$$

We have not calculated the integrals appearing in higher-particle intermediate state contributions.

\section{Acknowledgments}

H.B. was supported partially by the grant Volkswagenstiftung within in the project "Nonperturbative aspects of quantum field theory in various space-time dimensions". H.B. also acknowledges to ICTP condensed matter group for hospitality, where part of this work was done. A.F. acknowledges support from PRONEX under contract CNPq 66.2002/1998-99 and $\mathrm{CNPq}$ (Conselho Nacional de Desenvolvimento Científico e Tecnológico). This work is also supported by the EU network EUCLID, 'Integrable models and applications: from strings to condensed matter', HPRN-CT-2002-00325.

[1] Heisenberg W., Der mathematische Rahmen der Quantentheorie der Wellenfelder, Zeitschrift für Naturforschung, 1946, V.1, 608-622.

[2] Chew G., The S-matrix theory of strong interaction, New York, W.A. Benjamin Inc., 1961.

[3] Schroer B., Truong T.T., Weisz P., Towards an explicit construction of the sine-Gordon theory, Phys. Lett. B, 1976, V.63, 422-424.

[4] Karowski M., Thun H.J., Truong T.T., Weisz P., On the uniqueness of a purely elastic S matrix in $1+1$ dimensions, Phys. Lett. B, 1977, V.67, 321-322.

[5] Karowski M., Thun H.J., Complete S matrix of the massive Thirring model, Nuclear Phys. B, 1977, V.130, 295-308.

[6] Zamolodchikov A.B., Zamolodchikov A.B., Factorized S-matrices in two dimensions as the exact solutions of certain relativistic quantum field models, Annals Phys., 1979, V.120, 253-291. 
[7] Berg B., Karowski M., Weisz, P., Construction of Green functions from an exact S matrix, Phys. Rev. D, 1979, V.19, 2477-2479.

[8] Karowski M., Weisz P., Exact form factors in (1+1)-dimensional field theoretic models with soliton behavior, Nuclear Phys. B, 1978, V.139, 455-476.

[9] Smirnov F.A., Form factors in completely integrable models of quantum field theory, Adv. Series in Math. Phys., Vol. 14, World Scientific, 1992.

[10] Babujian H.M., Fring A., Karowski M., Zapletal A., Exact form factors in integrable quantum field theories: the sine-Gordon model, Nuclear Phys. B, 1999, V.538, 535-586, hep-th/9805185.

[11] Babujian H., Foerster A., Karowski M., The nested $S U(N)$ off-shell Bethe ansatz and exact form factors, J. Stat. Mech., 2006, to appear, hep-th/0611012.

[12] Babujian H., Karowski M., Exact form factors for the scaling $Z(N)$-Ising and the affine $A(N-1)$ Toda quantum field theories, Phys. Lett. B, 2003, V.575, 144-150, hep-th/0309018.

[13] Babujian H., Foerster A., Karowski M., Exact form factors in integrable quantum field theories: the scaling $Z(N)$-Ising model, Nuclear Phys. B, 2006, V.736, 169-198, hep-th/0510062.

[14] Babujian H.M., Correlation function in WZNW model as a Bethe wave function for the Gaudin magnetics, in Gosen 1990, Proceedings, Theory of Elementary Particles, 1990, 12-23 (see High Energy Physics Index, 1991, V.29, N 12257).

[15] Babujian H.M., Off-shell Bethe ansatz equation and $N$ point correlators in $S U(2)$ WZNW theory, J. Phys. A: Math. Gen., 1993, V.26, 6981-6990.

[16] Babujian H.M., Flume R., Off-shell Bethe ansatz equation for Gaudin magnets and solutions of KnizhnikZamolodchikov equations, Modern Phys. Lett. A, 1994, V.9, 2029-2040, hep-th/9310110.

[17] Frenkel I.B., Reshetikhin N.Y., Quantum affine algebras and holonomic difference equations, Comm. Math. Phys., 1992, V.146, 1-60.

[18] Schechtman V., Varchenko A., Arrangements of hyerplanes and Lie algebra homology, Invent. Math., 1991, V.106, 139-194.

[19] Bariev R.Z., On the rotational symmetry of the spin correlation function of the two-dimensional Ising model, Phys. Lett. A, 1976, V.55, 456-458.

[20] McCoy B., Tracy C.A., Wu T.T., Two-dimensional Ising model as an exactly solvable relativistic quantum field theory: explicit formulas for n point functions, Phys. Rev. Lett., 1977, V.38, 793-796.

[21] Sato M., Miwa T., Jimbo M., Studies on holonomic quantum fields: I, Proc. Japan Acad. A, 1977, V.53, 6-146.

[22] Yurov V.P., Zamolodchikov Al.B., Truncated fermionic space approach to the critical 2-D Ising model with magnetic field, Internat. J. Modern Phys. A, 1991, V.6, 4557-4578.

[23] Babujian H., Karowski M., Towards the construction of Wightman functions of integrable quantum field theories, Internat. J. Modern Phys. A, 2004, V.19S2, 34-49, hep-th/0301088.

[24] Smirnov F.A., private communication.

[25] Lechner G., An existence proof for interacting quantum field theories with a factorizing S-matrix, math-ph/0601022.

[26] Schroer B., Modular localization and the bootstrap-formfactor program, Nuclear Phys. B, 1997, V.499, 547-568, hep-th/9702145.

[27] Schroer B., Modular wedge localization and the $d=1+1$ formfactor program, Annals Phys., 1999, V.275, 190-223, hep-th/9712124.

[28] Schroer B., Wiesbrock H.W., Modular constructions of quantum field theories with interactions, Rev. Math. Phys., 2000, V.12, 301-326, hep-th/9812251.

[29] Korepin V.E., Oota T., A determinant representation for a correlation function of the scaling Lee-Yang model, J. Phys. A: Math. Gen., 1998, V.31, L371-L380, hep-th/9802003.

[30] Korepin V.E., Slavnov N.A., The determinant representation for quantum correlation functions of the sinhGordon model, J. Phys. A: Math. Gen., 1998, V.31, 9283-9295, hep-th/9801046.

[31] Karowski M., On the bound state problem in $(1+1)$ dimensional field theories, Nuclear Phys. B, 1979, V.153, 244-252.

[32] Babujian H., Karowski M., Exact form factors in integrable quantum field theories: the sine-Gordon model (II), Nuclear Phys. B, 2002, V.620, 407-455, hep-th/0105178.

[33] Berg B., Karowski M., Kurak V., Weisz P., Factorized $U(n)$ symmetric S-matrices in two dimensions, Nuclear Phys. B, 1978, V.134, 125-132. 
[34] Kurak V., Swieca J.A., Anti-particles as bound states of particles in the factorized S-matrix framework, Phys. Lett. B, 1979, V.82, 289-291.

[35] Köberle R., Swieca J. A., Factorizable $Z(N)$ models, Phys. Lett. B, 1979, V.86, 209-210.

[36] Köberle R., Kurak V., Swieca J.A., Scattering theory and 1/N expansion in the chiral Gross-Neveu model, Phys. Rev. D, 1979, V.20, 897-902.

[37] Berg B., Weisz P., Exact S-matrix of the chiral invariant $S U(N)$ Thirring model, Nuclear Phys. B, 1979, V.146, 205-214.

[38] Abdalla E., Berg B., Weisz P., More about the $S$-matrix of the chiral $S U(N)$ Thirring model, Nuclear Phys. B, 1979, V.157, 387-391.

[39] Belavin A.A., Exact solution of the two-dimensional model with asymptotic freedom, Phys. Lett. B, 1979, V.87, 117-121.

[40] Lehmann H., Symanzik K., Zimmermann W., On the formulation of quantized field theories, Nuovo Cimento, 1955, V.1, 205-225.

[41] Karowski M., The bootstrap program for $1+1$ dimensional field theoretic models with soliton behavior, in Field Theoretic Methods in Particle Physics, Editor W. Rühl, New York, Plenum Pub. Co., 1980, 307-324.

[42] Watson K.M., Some general relations between the photoproduction and scattering of pi mesons, Phys. Rev., 1954, V.95, 228-236.

[43] Nakayashiki A., Takeyama Y., On form factors of $S U(2)$ invariant Thirring model, in MathPhys Odyssey 2001, Integrable Models and Beyond - in Honor of Barry M. McCoy, Editors M. Kashiwara and T. Miwa, Progr. in Math. Phys., Birkäuser, 2002, 357-390, math-ph/0105040.

[44] Takeyama Y., Form factors of $S U(N)$ invariant Thirring model, Publ. Res. Inst. Math. Sci. Kyoto, 2003, V.39, 59-116, math-ph/0112025.

[45] Babujian H., Karowski M., Sine-Gordon form factors and quantum field equations, J. Phys. A: Math. Gen., 2002, V.35, 9081-9104, hep-th/0204097.

[46] Fring A., Mussardo G., Simonetti P., Form-factors for integrable Lagrangian field theories, the sinh-Gordon theory, Nuclear Phys. B, 1993, V.393, 413-441, hep-th/9211053.

[47] Koubeck A., Mussardo G., On the operator content of the sinh-Gordon model, Phys. Lett. B, 1993, V.311, 193-201.

[48] Brazhnikov V., Lukyanov S., Angular quantization and form factors in massive integrable models, Nuclear Phys. B, 1998, V.512, 616-636, hep-th/9707091.

[49] Babujian H., Karowski M., The exact quantum sine-Gordon field equation and other non-perturbative results, Phys. Lett. B, 1999, V.411, 53-57, hep-th/9909153.

[50] Smirnov F.A., Reductions of the sine-Gordon model as a perturbation of minimal models of conformal field theory, Nuclear Phys. B, 1990, V.337, 156-180.

[51] Zamolodchikov A.B., Irreversibility of the flux of the renormalization group in a 2-D field theory, JETP Lett., 1986, V.43. 730-732.

[52] Cardy J.L., The central charge and universal combinations of amplitudes in two-dimensional theories away from criticality, Phys. Rev. Lett., 1988, V.60, 2709-2711.

[53] Lukyanov S., Zamolodchikov A.B., Exact expectation values of local fields in quantum sine-Gordon model, Nuclear Phys. B, 1997, V.493, 571-587, hep-th/9611238.

[54] Babujian H., Karowski M., Short distance behavior of integrable quantum field theories and exact Wightman functions - summation over all intermediate states, in preparation. 